\title{
Effective Kerr Nonlinearity and Two-Color Solitons in Photonic Band-Gap Fibers Filled with a Raman Active Gas
}

\author{
D.V. Skryabin, F. Biancalana, D. M. Bird, and F. Benabid \\ Department of Physics, University of Bath, Bath BA2 7AY, United Kingdom
}

(Received 27 April 2004; published 30 September 2004)

\begin{abstract}
We predict a strong effective Kerr nonlinearity in hollow-core photonic crystal fibers filled with a Raman active gas which exceeds the intrinsic Kerr nonlinearity by 2 orders of magnitude. Two-color bright-bright and dark-bright solitons supported by this nonlinearity are found and the feasibility of their experimental observation is demonstrated.
\end{abstract}

DOI: 10.1103/PhysRevLett.93.143907

The guiding of light using photonic crystals fibers (PCFs) is becoming increasingly important in science and technology [1,2]. Among the many opportunities offered by PCFs, one of the most promising, and having implications across many disciplines, is the control and enhancement of nonlinear optical processes $[1,3,4]$. For example, gas filled hollow-core PCFs can provide an enhancement of the efficiency of nonlinear frequency conversion by many orders of magnitude $[3,4]$. In particular, the threshold for stimulated Raman scattering in hydrogen-filled PCFs has been already lowered by 6 orders of magnitude [3] and the efficiency of four-wave mixing has been increased by more than two orders [4] compared to conventional methods. Another promising and overlapping research direction concerns solitonic regimes of light propagation in PCFs [5,6]. Solitary waves, or simply solitons, are self-sustained localized wave packets which are attracting much interest not only in optics but also in many other diverse areas [7]. The potential to control the nonlinear and dispersive properties of PCFs provides fascinating opportunities to challenge new frontiers in this field also $[5,6]$.

The problem of controlling nonlinearities has been tackled well before the first PCF emerged from the fiber drawing tower. A well known example of an artificial nonlinearity is the effective or cascaded Kerr nonlinearity induced by the second harmonic in materials with quadratic $\chi^{(2)}$ nonlinearity [8,9]. Another example of a similar nonlinearity occurs in Raman active materials which are driven by two fields with the frequency difference detuned from the frequency of the Raman transition $[10,11]$. The strength of the effective nonlinearities can be orders of magnitude larger than the intrinsic Kerr nonlinearity. An important feature of the effective nonlinearities is that they are based on nonlinear interaction of two or more frequency components. Therefore if one wants to gain the advantages of solitonic regimes of propagation, then the problem of the existence and stability of inherently coupled multifrequency solitons should be addressed. Such solitons due to $\chi^{(2)}$ nonlinearities have been comprehensively analyzed recently [9]. Here we suggest the use of a hollow-core PCF filled with a
PACS numbers: 42.81.Dp, 42.65.Dr, 42.65.Tg, 42.70.Qs

Raman active gas pumped by two-color pulses for creating an effective nonlinearity which exceeds the intrinsic Kerr nonlinearity of such fibers by 2 orders of magnitude. We propose to use this system for the observation of various two-color temporal solitons at peak powers of order $10 \mathrm{~kW}$, which is well below the megawatt peak powers needed to observe solitons due to intrinsic nonlinearities [5].

The use of short pulses for inducing effective nonlinearity is preferred over the use of a continuous wave [11], due to the power limitations of the latter. The danger here, however, is that pulses propagating at different group velocities will simply walk through one another, quickly destroying the induced nonlinearity. However, as we show below, photonic band-gap fibers offer unique opportunities to match the group velocities of the fundamental fiber modes and even adjust the fiber dispersion to achieve the matching for the required frequencies. It is also important that the group velocity matching in these fibers can take place when the group velocity dispersions (GVDs) of the interacting fields have the same sign. This creates an ideal environment for the observation of temporal bright-bright solitons [12].

We consider the PCF shown in Figs. 1(b) and 1(c) with the distance between the centers of the air holes, i.e., pitch, equal to $\Lambda$ and the strut thickness $0.03 \Lambda$. This

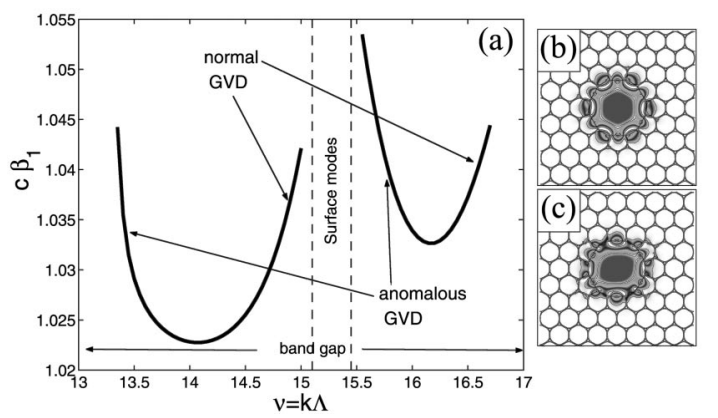

FIG. 1. (a) Ratio of the speed of light in vacuum to the group velocity of the fundamental PCF mode versus $k \Lambda$. Fundamental mode of the PCF for (b) $k \Lambda=14.828$ and (c) $k \Lambda=16.337$. 
structure is very similar to the PCFs used in experiments [2,5]. Dispersion of the PCF modes can be characterized by the dependence of $\beta \Lambda$ on $\nu=k \Lambda$, where $\beta(\omega)$ is the propagation constant, $k$ is vacuum wave number, $\omega=$ $c \nu / \Lambda$ is the angular frequency, and $c=3 \times 10^{8} \mathrm{~m} / \mathrm{s}$. The group velocity is given by $1 / \beta_{1}$ [see Fig. 1(a)], where $\beta_{1}=\partial_{\omega} \beta=c^{-1} d(\beta \Lambda) / d \nu$. An important feature of the PCF under consideration and of the ones used in [2,5] is the presence of one or more high loss spectral windows where the dynamics is dominated by the surface modes localized around the walls of the fiber core; see [2] and Fig. 1(a). As a result of this, the photonic band gap has several branches of the fundamental modes and each branch has a point where the GVD, $\beta_{2}=\partial_{\omega} \beta_{1}$, is zero. Clearly, the dispersion characteristic in Fig. 1(a) offers several opportunities to match the group velocities at the two frequencies. Moreover, if the group velocities at $\nu_{1}$ and $\nu_{2}$ are matched, then the corresponding frequency difference is given by $\Delta=c\left(\nu_{1}-\nu_{2}\right) / \Lambda$ and a fiber with the pitch required to achieve the group velocity matching for a given $\Delta$ can be fabricated.

We consider the above PCF filled with a Raman active gas and pumped by two pulses with a frequency difference $\Delta=\left(\omega_{1}-\omega_{2}\right)>0$ detuned from the frequency $\omega_{r}$ of the Raman resonance, i.e., $\omega_{r}-\Delta \neq 0$. Dimensionless equations describing this process are

$$
\begin{gathered}
i \partial_{z} E_{1}=i v \partial_{t} E_{1}-d_{1} \partial_{t}^{2} E_{1}-\kappa E_{2} Q, \\
i \partial_{z} E_{2}=-i v \partial_{t} E_{2}-d_{2} \partial_{t}^{2} E_{2}-\kappa E_{1} Q^{*}, \\
0=i \partial_{t} Q+(\delta+i \epsilon) Q+\kappa E_{1} E_{2}^{*} .
\end{gathered}
$$

Equations (1)-(3) describe the simplest situation, when the Raman transition is not saturated and energy transfer to the higher-order Stokes and anti-Stokes components either does not happen or can be disregarded [11,13]. $Q$ is the Raman coherence and $\kappa$ is the dimensionless coupling constant, the choice of which is determined by the choice of the normalization of the slowly varying amplitudes $E_{1,2}$ and $Q$. Dimensionless delay $t$ is measured in the units of the characteristic pulse duration $\tau$, and the propagation distance $z$ is in the units of the GVD length $l_{g v d}=\tau^{2} /$ $\left|\beta^{(2)}\left(\omega_{2}\right)\right|$. Then $d_{2}=\operatorname{sgn}\left[\beta^{(2)}\left(\omega_{2}\right)\right]$ and $d_{1}=\beta^{(2)}\left(\omega_{1}\right) /$ $\left|\beta^{(2)}\left(\omega_{2}\right)\right|$ is the ratio of the GVDs of the fields. $\delta$ is the normalized detuning of the frequency difference of the driving fields from the Raman resonance frequency $\omega_{r}$, i.e., $\delta=\tau\left(\omega_{r}-\Delta\right) . \epsilon=\tau / T_{2}$, where $1 / T_{2}$ is the width of the Raman resonance. The parameter $v$ characterizes the difference of the group velocities of the driving fields and it is expressed as half of the ratio of the GVD length and of the walk-off length $l_{\text {gvm }}=\tau /\left[\beta^{(1)}\left(\omega_{2}\right)-\beta^{(1)}\left(\omega_{1}\right)\right]$ : $v=l_{\text {gvd }} /\left(2 l_{\text {gvm }}\right)$.

Throughout this work, we consider the situation of a large Raman detuning: $|\delta| \gg 1$ and $|\delta| \gg \epsilon$. Then by neglecting $\epsilon$ we derive an approximate solution for the coherence

$$
Q=-\frac{\kappa}{\delta}\left[1-\frac{i}{\delta} \partial_{t}+O\left(|\delta|^{-2}\right)\right] E_{1} E_{2}^{*}
$$

By substituting (4) into Eqs. (1) and (2), one can derive nonlinearly coupled Schrödinger equations. An important property of these equations is that they do not have nonlinear self-phase modulation terms [14]. For $\delta>0$ the effective nonlinearity is focusing and for $\delta<0$ it is defocusing. If one compares Eqs. (1)-(3) with the system used to study temporal Raman solitons in gas cells [13], then the difference is that $v=d_{1,2}=\delta=0$ in the latter, which makes the physical origin and properties of the solitons described in [13] completely different from our case.

It is convenient here to adopt the standard normalization used in the fiber optics, when physical fields are normalized to the square root of the so-called nonlinear fiber parameter $\gamma$ [14]. This is achieved by choosing $\kappa=$ $|\delta|^{1 / 2}$. Let us now derive an expression for $\gamma_{\text {eff }}$, which is the nonlinear parameter due to the effective Kerr nonlinearity. One can verify that $|\delta| /\left(\delta^{2}+\epsilon^{2}\right)=C \gamma_{\text {eff }}$, where $C$ is some proportionality coefficient. Using the definition of the steady-state Raman gain at the line center, $g_{\text {ss }}$ (see [13]), we find that $\epsilon /\left(\delta^{2}+\epsilon^{2}\right)=$ $C g_{\text {ss }} / A /\left(1+T_{2}^{2}\left|\omega_{r}-\Delta\right|^{2}\right)$, where $A$ is the area of the fiber mode. In the limit of a large detuning from the Raman resonance the last two expressions give

$$
\gamma_{\mathrm{eff}} \simeq \frac{g_{\mathrm{ss}}}{A} \frac{1}{T_{2}\left|\omega_{r}-\Delta\right|} .
$$

We now turn our attention to the soliton solutions of Eqs. (1), (2), and (4). By analogy with other two component systems [14] we expect that these solitons can be parametrized by three independent parameters associated with invariances with respect to the two phase rotations, $E_{1,2} \rightarrow E_{1,2} e^{i \psi_{1,2}}$ and translations of $t$. We neglect terms of $O\left(|\delta|^{-2}\right)$ in Eq. (4) and seek solitons of the form $E_{1,2}=f_{1,2}(\xi) e^{-i q_{1,2} z}$, where $\xi=\tau-V z$ and $V$ is a parameter [9]. We have solved the system of ordinary differential equations for the functions $f_{1,2}$ numerically and have identified the existence of three distinct soliton families: bright-bright, bright-dark, and dark-dark soliton pairs. Below we analyze only the bright-bright and bright-dark solitons.

Bright-bright solitons are likely to be the easiest to excite and observe in future experiments. They can exist either when the GVDs at both frequencies are anomalous $\left(d_{1,2}<0\right)$ and the effective nonlinearity is focusing $(\delta>$ $0)$ or when the GVDs are normal $\left(d_{1,2}>0\right)$ and the nonlinearity is defocusing $(\delta<0)$. The decay rates of the tails of the bright-bright solitons are given by $\lambda_{1,2}=$ $\sqrt{4 q_{1,2}\left|d_{1,2}\right|-(v \pm V)^{2}} /\left|2 d_{1,2}\right|$ and the solitons exist providing $\operatorname{Im} \lambda_{1,2}=0$. Linking parameters $q_{1,2}$ and $V$ by the condition that the tails of both components decay with the same rate, i.e., $\lambda_{1}=\lambda_{2}$, and taking $Q=-s E_{1} E_{2}^{*} / \kappa$, 
where $s=\operatorname{sgn}(\delta)$, we have found the explicit form of the bright-bright solitons:

$$
E_{1,2}=a_{1,2} \exp \left\{i \xi \frac{V \pm v}{2 d_{1,2}}-i q_{1,2} z\right\} \operatorname{sech} \frac{\xi}{w} .
$$

Here $w=\sqrt{-2 s d_{2}} / a_{1}, a_{2}=a_{1} \sqrt{d_{1} / d_{2}}$, and

$$
a_{1}^{2}=\frac{2\left|d_{2}\right|}{\left|d_{1}\right|}\left\{s q_{1}-\frac{(V+v)^{2}}{4\left|d_{1}\right|}\right\} .
$$

For (6) to be a good approximate solution to the exact system (1)-(3), we require that $\left|\partial_{t} E_{1}^{*} E_{2}\right| \ll|\delta|$. This condition ensures that the second term in the expansion (4) is much less than the first and that it is satisfied for the soliton (6) if

$$
\left|d_{2}(V+v)-d_{1}(V-v)\right| \ll 2\left|\delta d_{1} d_{2}\right| .
$$

Also it is clear at the intuitive level that if the walk-off length $l_{\text {gvm }}$ becomes equal to or smaller than $l_{g v d}$, then it can greatly complicate the practical observation of the two-color solitons, because the two pulses will tend to separate spatially quicker than GVD can be compensated by nonlinearity. As is clear from Fig. 1(a), the matching of group velocities for two fields having GVDs of the same sign is possible only between the two branches of the fundamental mode.

From the Raman active gases, we have chosen $\mathrm{SF}_{6}$ as our working example. $\mathrm{SF}_{6}$ has $\omega_{r} \simeq 2 \pi \times 23 \mathrm{THz}$, which easily fits into the photonic band gap for typical values of $\Lambda$. The gain coefficient for $\mathrm{SF}_{6}$ is $g_{\mathrm{ss}} \simeq 14 \times 10^{-14} \mathrm{~m} / \mathrm{W}$. Recent experiments with $\mathrm{SF}_{6}$, where the symmetric vibrational Raman mode has been excited in hollow waveguides [15], have shown values of $T_{2}$ of order several picoseconds. Choosing $\tau=1 \mathrm{ps}, T_{2}=1 \mathrm{ps}$, and detuning from the resonance $\omega_{r}-\Delta \equiv \alpha / T_{2}$, where $\alpha$ is a number, we find that $\delta=\alpha$ and $\epsilon=1$. For example, taking $\Delta=$ $2 \pi \times 24.6 \mathrm{THz}$ gives $\alpha \simeq-10$ and we have $|\delta| \gg 1$, $|\delta| \gg \epsilon$. Negative values of $\delta$ imply defocusing nonlinearity and require normal GVDs for the bright solitons to exist. Choosing, for example, $\lambda_{1}=1.2712 \mu \mathrm{m}$, we achieve group velocity matching with the second pulse at $\lambda_{2}=1.1538 \mu \mathrm{m}$ for $\Lambda=3 \mu \mathrm{m}$. These $\Lambda / \lambda_{1,2}$ values correspond to the modes shown in Figs. 1(b) and 1(c). Corresponding values of $\beta_{2}$ are $1.2 \mathrm{ps}^{2} / \mathrm{m}$ for $\lambda_{1}$ and $0.4 \mathrm{ps}^{2} / \mathrm{m}$ for $\lambda_{2}$. Despite the fact that we are detuned by ten linewidths from the resonance, we have $\gamma_{\text {eff }} \simeq$ $10^{-4} \mathrm{~W}^{-1} \mathrm{~m}^{-1}$, which is 2 orders of magnitude larger than the value of $\gamma$ due to intrinsic Kerr nonlinearities: $\gamma_{\text {intr }} \simeq 10^{-6} \mathrm{~W}^{-1} \mathrm{~m}^{-1}$ [5]. Thus our neglecting the intrinsic Kerr nonlinearity, which otherwise would give selfphase modulation terms in Eqs. (1) and (2), is well justified for the conditions discussed in this work. Also it follows that the observation of solitons due to effective nonlinearity requires peak powers of order $10 \mathrm{~kW}$ only, and not of order $1 \mathrm{MW}$ as in [5].
One of the prime conditions for observation of solitons is their robustness against the growth of small perturbations. We have studied the stability of bright-bright solitons using the linearization of Eqs. (1), (2), and (4) and their direct numerical simulation and have not found any instabilities, though the range of parameters is too wide to completely eliminate their existence. The existence and stability of these solitons within the full model (1)-(3) is a more delicate problem, though. Assuming $\epsilon=0$ and solving Eqs. (1)-(3) numerically with the initial conditions (6), we have found that, if the group velocities are exactly matched, then these approximate solitons quickly adjust to the full model and propagate without disturbance for many $l_{g v d}$; see Figs. 2(a)-2(c). Small values of the parameter $v$, ensuring that the condition (8) is well satisfied, also do not alter the stability of the solitons. However, introducing a moderate mismatch of the group velocities, which starts to endanger condition (8), leads to a strong outburst of material excitation and destruction of the soliton; see Figs. 2(d)-2(f). Solving Eqs. (1)-(3) with $\epsilon=1$, we have observed the same stable and unstable scenarios of the soliton evolution, which have been accompanied by the small energy flow from the pump $E_{1}$ to the Stokes wave $E_{2}$. However, the effect of this flow does not change the solitons significantly even for long propagation distances of tens of meters.

Dark-bright solitons exist when the GVDs at the two frequencies have opposite signs. For the sake of concreteness we assume the GVD to be normal for $E_{1}$, i.e., $d_{1}>0$, and anomalous for $E_{2}$, i.e., $d_{2}<0$. Then we have found that the three-parameter family of dark-bright solitons

$$
\begin{gathered}
E_{1}=A_{1} \exp \left\{i \xi \frac{V+v}{2 d_{1}}-i q_{1} z\right\} \operatorname{sech} \frac{\xi}{\tilde{w}}, \\
E_{2}=A_{2} \exp \left\{i \Omega_{2} \xi-i q_{2} z\right\}\left[\cos \phi \tanh \frac{\xi}{\tilde{w}}+i \sin \phi\right], \\
A_{1}^{2}=\frac{a_{1}^{2} \cos ^{2} \phi}{1+\sin ^{2} \phi}, \quad A_{2}^{2}=\frac{\left|d_{1}\right|}{\left|d_{2}\right|} \frac{a_{1}^{2}}{\left(1+\sin ^{2} \phi\right)}
\end{gathered}
$$

exists providing that the effective nonlinearity is focussing, i.e., $\delta>0, s=1$. Here $q_{2}=(v-V) \Omega_{2}+d_{2} \Omega_{2}^{2}$, $\tilde{w}=\sqrt{-2 d_{2}} / A_{1}$, and $\Omega_{2}=\left(\tilde{w} A_{1}^{2}-v+V\right) /\left(2 d_{2}\right)$. In Eq. (10), $\phi$ is an angle characterizing the depth of the notch in the dark component. The solution (9)-(11) is parametrized by $q_{1}, V$, and $\phi$, which are not linked by any condition.

These types of solitons are called inverted dark-bright solitons [16], because their dark component, which is naturally expected at normal GVD, happens to be at anomalous GVD, while the bright component occurs at normal GVD. Conventional, i.e., not inverted, dark-bright solitons have attracted significant attention. The inverted dark-bright solitons have so far generated much less interest [16]. This is because the background of the dark 

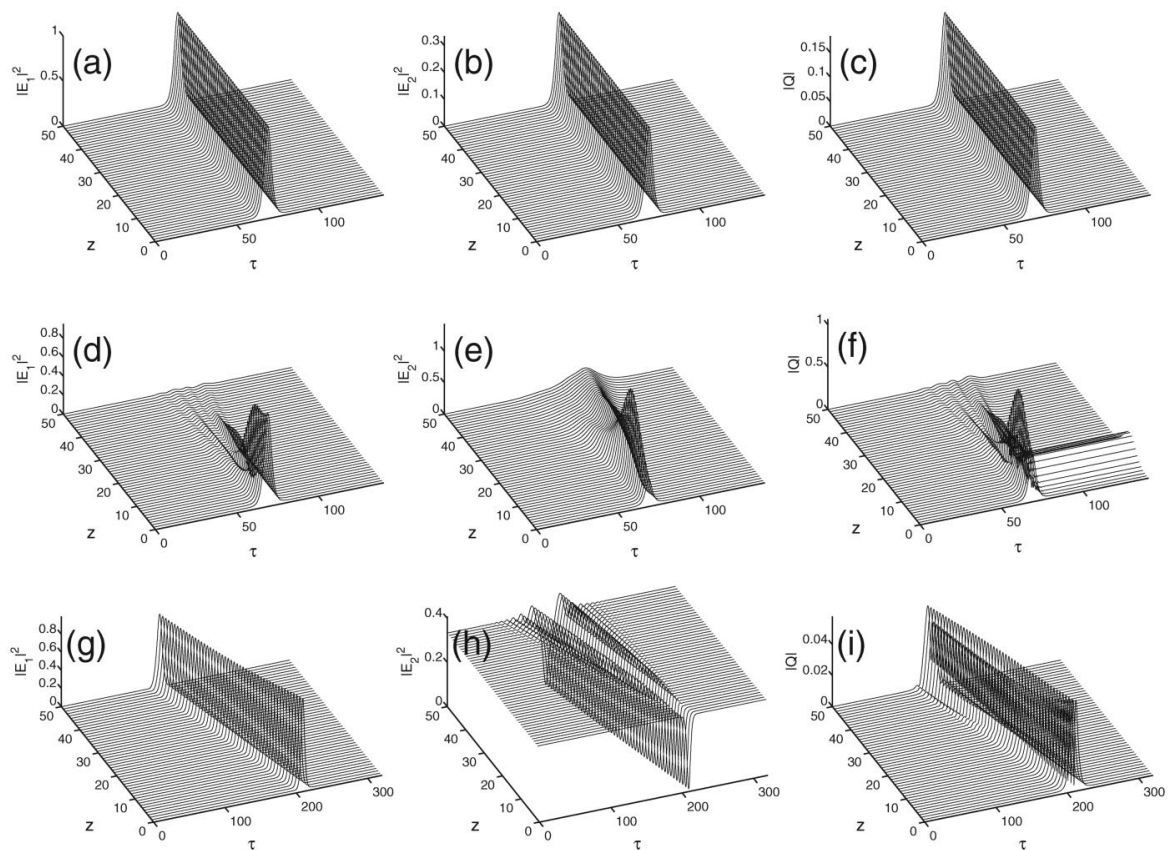

FIG. 2. Numerical modeling of Eqs. (1)-(3) with solitonic initial conditions (6) and (9)(11). (a) -(c) Bright-bright soliton: $\kappa=\sqrt{10}, d_{1}=1, d_{2}=3$, $\delta=-10, \quad s=-1, \quad q_{1}=10$, $v=V=0$. (d)-(f) The same as (a)-(c) but for $v=3$. (g)(i) Inverted dark-bright soliton: $\kappa=\sqrt{10}, \quad d_{1}=1, \quad d_{2}=-3$, $\delta=10, s=1, v=3, q_{1}=10$, $\phi=V=0$. part of the inverted solitons, supported by intrinsic Kerr nonlinearity, is modulationally unstable due to the combined action of the focusing self-phase modulation and anomalous GVD [16]. In our case, however, the self-phase modulation is absent or negligible compared to the crossphase modulation, which ensures the stability of the background of the dark component. Stability properties of the inverted dark-bright solitons in our model are similar to the properties discussed above for the brightbright solitons. Namely, when condition (8) holds firmly, we have observed undisturbed propagation for hundreds of $l_{\text {gvd }}$. This is valid for the full model (1)-(3) as well as when $Q$ is replaced by (4). When $v$ is increased, the full model starts to demonstrate distortions of the dark-bright solitons [see Figs. 2(g)-2(i)] induced by the spatial separation of the centers of the two fields. Note that matching of the group velocities for the dark-bright solitons can be achieved using either two frequencies on a single branch of the fundamental mode or between the two branches; see Fig. 1(a).

In summary, we have predicted strong effective Kerr nonlinearity induced by off-resonant Raman transitions in gas filled hollow-core PCFs, which can exceed the intrinsic Kerr nonlinearity by 2 orders of magnitude. We have also demonstrated the feasibility of experimental observation of bright-bright and dark-bright temporal solitons supported by the effective nonlinearity.

We acknowledge discussions with J.C. Knight and P. St. J. Russell.

[1] P. St. J. Russell, Science 299, 358 (2003).

[2] C. M. Smith et al., Nature (London) 424, 657 (2003).
[3] F. Benabid et al., Phys. Rev. Lett. 93, 123903 (2004).

[4] S. O. Konorov, A. B. Fedotov, and A. M. Zheltikov, Opt. Lett. 28, 1448 (2003).

[5] D. G. Ouzounov et al., Science 301, 1702 (2003).

[6] J. Herrmann et al., Phys. Rev. Lett. 88, 173901 (2002); W. H. Reeves et al., Nature (London) 424, 511 (2003); D. V. Skryabin, F. Luan, J. C. Knight, and P. St. J. Russell, Science 301, 1705 (2003).

[7] A. Scott, Nonlinear Science: Emergence \& Dynamics of Coherent Structures (Oxford University Press, Oxford, 1999).

[8] For a review, see G. I. Stegeman, Quantum Semiclass. Opt. 9, 139 (1997).

[9] For a review, see A.V. Buryak, P. Di Trapani, D.V. Skryabin, and S. Trillo, Phys. Rep. 370, 63 (2002).

[10] S. E. Harris and A.V. Sokolov, Phys. Rev. Lett. 81, 2894 (1998); for a review, see A.V. Sokolov and S. E. Harris, J. Opt. B 5, 1 (2003).

[11] D. D. Yavuz, D. R. Walker, and M. Y. Shverdin, Phys. Rev. A 67, 041803 (2003).

[12] A complicated experimental technique of dispersion manipulation, leading to the formation of temporal two frequency solitons in bulk quadratically nonlinear crystals, is described in P. Di Trapani et al., Phys. Rev. Lett. 81, 570 (1998).

[13] K. J. Druhl, R. G. Wenzel, and J. L. Carlsten, Phys. Rev. Lett. 51, 1171 (1983); J. Reintjes and M. Bashkansky, in Handbook of Optics, edited by M. Bass (McGraw-Hill, New York, 2001), Vol. IV, Chap. 18.

[14] Y.S. Kivshar and G. P. Agrawal, Optical Solitons (Academic Press, San Diego, 2003).

[15] M. Wittman, A. Nazarkin, and G. Korn, Phys. Rev. Lett. 84, 5508 (2000).

[16] S. Trillo, S. Wabnitz, E. M. Wright, and G. I. Stegeman, Opt. Lett. 13, 871 (1988). 